\title{
NEURAL NETWORKS AND SYSTOLIC ARRAY DESIGN
}




\section{SERIES IN MACHINE PERCEPTION AND ARTIFICIAL INTELLIGENCE*}

Editors: H. Bunke (Univ. Bern, Switzerland)

P. S. P. Wang (Northeastern Univ., USA)

Vol. 34: Advances in Handwriting Recognition

(Ed. S. W. Lee)

Vol. 35: Vision Interface - Real World Applications of Computer Vision

(Eds. M. Cheriet and Y.-H. Yang)

Vol. 36: Wavelet Theory and Its Application to Pattern Recognition

(Y. Y. Tang, L. H. Yang, J. Liu and H. Ma)

Vol. 37: Image Processing for the Food Industry

(E. R. Davies)

Vol. 38: New Approaches to Fuzzy Modeling and Control - Design and Analysis (M. Margaliot and G. Langholz)

Vol. 39: Artificial Intelligence Techniques in Breast Cancer Diagnosis and Prognosis (Eds. A. Jain, A. Jain, S. Jain and L. Jain)

Vol. 40: Texture Analysis in Machine Vision (Ed. M. K. Pietikäinen)

Vol. 41: Neuro-Fuzzy Pattern Recognition (Eds. H. Bunke and A. Kandel)

Vol. 42: Invariants for Pattern Recognition and Classification (Ed. M. A. Rodrigues)

Vol. 43: Agent Engineering (Eds. Jiming Liu, Ning Zhong, Yuan Y. Tang and Patrick S. P. Wang)

Vol. 44: Multispectral Image Processing and Pattern Recognition (Eds. J. Shen, P. S. P. Wang and T. Zhang)

Vol. 45: Hidden Markov Models: Applications in Computer Vision (Eds. H. Bunke and T. Caelli)

Vol. 46: Syntactic Pattern Recognition for Seismic Oil Exploration (K. Y. Huang)

Vol. 47: Hybrid Methods in Pattern Recognition (Eds. H. Bunke and A. Kandel)

Vol. 48: Multimodal Interface for Human-Machine Communications (Eds. P. C. Yuen, Y. Y. Tang and P. S. P. Wang)

Vol. 49: Neural Networks and Systolic Array Design (Eds. D. Zhang and S. K. Pal) 


\section{NEURAL NETWORKS AND SYSTOLIC ARRAY DESIGN}

\section{Editors}

\section{D)}

Hong Kong Polytechnic University, Hong Kong

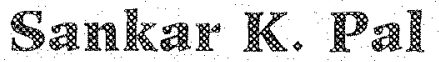

The Indian Statistical Institute, Calcutta, India 
Published by

World Scientific Publishing Co. Pte. Ltd.

P O Box 128, Farrer Road, Singapore 912805

USA office: Suite 1B, 1060 Main Street, River Edge, NJ 07661

UK office: 57 Shelton Street, Covent Garden, London WC2H 9HE

\section{British Library Cataloguing-in-Publication Data}

A catalogue record for this book is available from the British Library.

\section{NEURAL NETWORKS AND SYSTOLIC ARRAY DESIGN}

Copyright $(2002$ by World Scientific Publishing Co. Pte. Ltd.

All rights reserved. This book, or parts thereof, may not be reproduced in any form or by any means, electronic or mechanical, including photocopying, recording or any information storage and retrieval system now known or to be invented, without written permission from the Publisher.

For photocopying of material in this volume, please pay a copying fee through the Copyright Clearance Center, Inc., 222 Rosewood Drive, Danvers, MA 01923, USA. In this case permission to photocopy is not required from the publisher.

ISBN 981-02-4840-7 


\section{Preface}

In recent years, Artificial Neural Networks (ANNs) have become a subject of very extensive research, since they are envisioned as an alternative to solving problems of certain tasks such as pattern recognition, vision and speech recognition, where conventional artificial intelligence techniques had been unable to tackle efficiently. Neural networks are biologically inspired; they are composed of elements that perform in a manner analogous to the most elementary functions of biological neurons. However, as simulations of large neural networks on a sequential computer frequently require days and weeks of computations, and the long computational time had been a critical obstacle to the progress in neural network research, extensive effort is being devoted to the parallel implementation of neural networks. Systolic Arrays (SAs) offer some of the best solutions to the parallel implementation of neural networks. It can overcome the communication problems generated by the highly interconnected neurons, and can exploit the massive parallelism inherent in the problem. Moreover, since the computation model of neural networks can be represented by a series of matrix by vector multiplications, the classical systolic algorithms can be used to implement them.

SA design differs from that of the conventional von Neumann computer in its highly pipelined computation. Once a data item is brought out from the memory it can be used effectively at each cell it passes while being pumped from cell to cell along the array. This is especially appealing for a wide class of compute-bound computations, where multiple operations are performed on each data item in a repetitive manner. This avoids the classic memory access bottleneck 
problem commonly incurred in von Neumann machines. In addition, SA has a spatial locality and a temporal locality. It manifests a locally communicative interconnection structure, and there is at least one unit-time delay allocated so that signal transactions from one processing element to the next can be completed. When a large number of processors work together, communication becomes a significant problem. Therefore, regular and local communication in systolic arrays is advantageous.

ANNs and SAs have many similar features. The communication only between neighbor neurons in neural networks is similar to the spatial/temporal locality of systolic arrays. The highly interconnected structures of neural networks can be mapped onto the regular structure of a systolic array, and the massive parallelism can be exploited by the large number of processing elements of systolic arrays. Since SAs can overcome the communication problems generated by highly interconnected neurons, and can exploit the massive parallelism inherent in the problem, there have been several research efforts on developing systolic algorithms and systolic array structures to implement various ANNs. Attempts are made to develop a mapping approach, in which the systolic algorithms are partitioned and mapped onto the parallel computers, and a VLSI approach, in which VLSI systolic arrays dedicated to specific models are designed.

This book provides a collection of sixteen chapters containing tutorial articles and new material describing, in a unified way, the basic concepts, theories and characteristic features of integrating and formulating different facets of ANNs and SAs, with recent developments and significant applications. The articles, written by various experts from around the world, demonstrate the varied ways this integration can be made to design methodologies, algorithms and architectures, and implementations for ANN applications efficiently. With the exception of the first chapter's tutorial introduction to ANNs and SAs, each chapter provides exhaustive information on developments in the respective areas, keeping a cohesive character with other chapters. The approaches typically include the mapping of systolic algorithms for neural networks onto parallel computers and the design of VLSI systolic arrays dedicated to one or two specific models, besides describing some available systems and applications. 
They also provide a balanced mixture of both theory and implementation.

This book, which is unique in character, is useful to graduate students and researchers in computer science, electrical engineering, systems science and information technology not only as a reference, but also as a text book for some parts of the curriculum. The researchers and practitioners in industry and $R \& D$ laboratories working in the fields of system design, VLSI, parallel processing, control, pattern recognition, neural networks and vision will also benefit.

We would like to take this opportunity to thank all the contributors for agreeing to write for the book. We owe a vote of thanks to Ian Seldrup and Alan Pui of World Scientific, Singapore, for taking the initiative in bringing the volume out. The technical/software support provided by Adams Kong, Michael Wong, Henry Ko and Yunzhi Lin are also acknowledged. The project was initiated when Prof. S.K. Pal was working as a Visiting Professor in the department of computing, Hong Kong Polytechnic University, Hong Kong, during November-December 2000.

David Zhang and Sankar K. Pal

December 2001 
This page is intentionally left blank 


\section{Contents}

Preface $\quad$ v

Chapter 1 Neural Networks and Systolic Arrays:

Models and Integration

D. Zhang and S.K. Pal

1.1 Introduction

1.2 Neural Network Models 4

1.3 Typical ANN Architectures 8

1.4 Systolic Array Implementation in VLSI 9

1.5 Book Perspective 13

Chapter 2 Systolic Array Methodology for a Neural Model to Solve the Mixture Problem

R.M. Pérez, P. Martinez, A. Plaza and

P.L. Aguilar

2.1 Introduction

2.2 Algorithmic Method 25

2.3 VLSI Implementation by Systolic Arrays 28

2.4 Conclusions 44

Chapter 3 Morphological Endmember Identification and Its Systolic Array Design

P.L. Aguilar, A. Plaza, P. Martínez and R.M. Pérez 
3.1 Introduction

3.2 Overview of the Proposed Application 48

3.3 Methodology 50

3.4 Systolic Array Implementation $\quad 54$

3.5 Summary of Design 65

3.6 Conclusions $\quad 67$

Chapter 4 MANTRA I: A Systolic Array for Neural Computation

M.A. Viredaz and P. Ienne

4.1 Introduction

4.2 The GENES IV Systolic Array 73

4.3 The MANTRA I Machine 75

4.4 Programming the MANTRA I Machine 82

4.5 Performance Analysis $\quad 84$

$\begin{array}{lll}4.6 & \text { Future Evolution } & 87\end{array}$

$\begin{array}{lll}4.7 \text { Conclusion } & 89\end{array}$

Chapter 5 Mixed-Signal Neuro-Fuzzy Processor Implementations: Sequential Architectures and Circuit-Level Description

J. Madrenas and E. Alarcón

5.1 Introduction

5.2 Sequentiality Analysis

5.3 VLSI Circuits for Mixed-Signal Sequential Implementations

5.4 Case Study 1: Mixed-Signal Implementation of a Discrete-time Sequential Takagi-Sugeno Fuzzy Controller

5.5 Case Study 2: VLSI Design of a Flexible-Structure Sequential Mixed-Signal Neural Processor 
Chapter 6 CMAC Neural Networks and Systolic

Implementation

B.D. Liu, Y.H. Kuo and J.S. Ker

6.1 Introduction

6.2 Standard CMAC-Based Neural Networks

6.3 Higher-Order CMAC (BCMAC)

6.4 Weight Cell Address Generation 145

6.5 Systolic Architecture for Addressing Weight Cells 150

6.6 VHDL Modeling of the Systolic Implementation 154

6.7 Conclusions

Chapter 7 Quadrant Interlocking Factorization on Systolic and Wavefront Array Processors

M.P. Bekakos, O.B. Efremides and D.J. Evans

7.1 Introduction

7.2 The Butterfly Procedure for Solving Linear Systems 165

7.3 Multilayered Dewavefront Array Processor Meshes 170

7.4 Block Generalized WZ Factorization 176

7.5 Systolic Quadrant Interlocking Elimination - SQIE 183

7.6 Conclusions

Chapter 8 Systolic S.O.M. Neural Network for Hyperspectral Image Classification

P. Martínez, P.L. Aguilar, R.M. Pérez and A. Plaza

8.1 Introduction

8.2 SOM Neural Network for Hyperspectral Analysis 194

8.3 Topology of the Proposed Neural Network 196

8.4 SOM Training Algorithm 196

8.5 Systolic Algorithm 198

8.6 Conclusions 202 
Chapter 9 Optimizing and Learning Algorithm for Feedforward Neural Networks and Its Implementation by Systolic Array

P.B. Burgos

9.1 Introduction

9.2 Householder Reflections

9.3 Selection of the Optimal Hidden Structure

9.4 The Optimizing and Learning Algorithm

9.5 Implementation of OLA by Systolic Arrays 214

9.6 Experimental Results 220

9.7 Conclusions 226

Chapter 10 Parallel ANN Architecture for Fuzzy Patterns

229

D. Zhang and S.K. Pal

10.1 Introduction

10.2 Fuzzy Clustering Neural networks (FCN) Model

10.3 Parallel Architecture

10.4 Systolic Array Design

10.5 Conclusions

Chapter 11 Pipelined Systolic Arrays for Time-Delay Neural Networks

D. Zhang and S.K. Pal

11.1 Introduction

11.2 Window Computing Model

11.3 Pipelined Architecture: Processing Stages 257

11.4 Systolic Array Implementation

11.5 Speech Recognition Applications 264

11.6 Structure Analysis 268

11.7 Conclusions 274

Chapter 12 An Integrated Intelligent Classification Engine $\left(I^{2} \boldsymbol{C E}\right)$ for Biosignal Engineering

A.N. Kastania and M.P. Bekakos 
12.2 The Concept of a Neural Classifier 280

12.3 Biosignal Quality Issues $\quad 282$

12.4 Classificationi via Adaptive Logic Networks (ALNs) 283

12.5 Classifier Quality Assurance 287

12.6 Conclusions and Outlook 295

Chapter 13 Multiplierless Designs for Artificial Neural Networks H.K. Kwan

13.1 Introduction

13.2 Weight and Bias Adaptation $\quad 305$

13.3 Activation Functions 306

13.4 Optimal Architectures for MMFNN Designs 308

13.5 Mixed OPOT Optimal MMFNN Architecture for Discrete Input to Discrete Output Mapping Applications

13.6 Mixed QN Optimal MMFNN Architecture for Continuous Input to Continuous Output at Mapping Applications

13.7 Concluding Remarks

Chapter 14 A VLSI System for Intelligent Decision Making in Real-Time

N. Ranganathan and M.I. Patel

14.1 Introduction

14.2 Why VLSI for Intelligent Decision Making? 328

$\begin{array}{ll}14.3 \text { Background } & 329\end{array}$

14.4 RAPID System Architecture 330

14.5 Functional Interaction between the ANN and the FES 332

14.6 Hardware Architecture 334

14.7 VLSI Prototype $\quad 348$

$\begin{array}{ll}14.8 \text { Performance } & 349\end{array}$

14.9 Conclusions $\quad 350$ 
Chapter 15 Reconfigurable Hardware Systolic Array for Real-Time Compartmental Modeling of Large-Scale Artificial Nervous Systems M. Korkin

15.1 Introduction

15.2 CBM Hardware Architecture

15.3 Host Interface

15.4 CBM Software Tools

15.5 Conclusions

Chapter 16 Implementing and Mapping ANNs on Reconfigurable Mesh Massively Parallel Architectures

W.N. Li and J.J. Jenq

16.1 Introduction

16.2 Feed-Forward Networks: Back-Propagation Learning 376

16.3 Reconfigurable Mesh with Buses 377

16.4 First Mapping Method 383

16.5 Second Mapping Method 389

16.5 Discussions 393

$\begin{array}{ll}\text { Index } & 397\end{array}$

About the Editors 403 